\title{
Observational aspects of IMF draping-related magnetosheath accelerations for northward IMF
}

\author{
B. Harris ${ }^{1}$, C. J. Farrugia ${ }^{1}$, N. V. Erkaev ${ }^{2,3}$, and R. B. Torbert ${ }^{1}$ \\ ${ }^{1}$ Space Science Center and Department of Physics, University of New Hampshire, NH, USA \\ ${ }^{2}$ Institute for Computational Modeling, Russian Academy of Sciences, Krasnoyarsk, Russia \\ ${ }^{3}$ Siberian Federal University, Krasnoyarsk, Russia
}

Correspondence to: C. J. Farrugia (charlie.farrugia@unh.edu)

Received: 31 March 2013 - Revised: 1 July 2013 - Accepted: 28 August 2013 - Published: 22 October 2013

\begin{abstract}
Acceleration of magnetosheath plasma resulting from the draping of the interplanetary magnetic field (IMF) around the magnetosphere can give rise to flow speeds that exceed that of the solar wind $\left(V_{\mathrm{SW}}\right)$ by up to $\sim 60 \%$. Three case event studies out of 34 identified events are described. We then present a statistical study of draping-related accelerations in the magnetosheath. Further, we compare the results with the recent theory of Erkaev et al. (2011, 2012). We present a methodology to help distinguish draping-related accelerations from those caused by magnetic reconnection. To rule out magnetopause reconnection at low latitudes, we focus mainly on the positive $B_{z}$ phase during the passage of interplanetary coronal mass ejections (ICMEs), as tabulated in Richardson and Cane (2010) for 1997-2009, and adding other events from 2010. To avoid effects of high-latitude reconnection poleward of the cusp, we also consider spacecraft observations made at low magnetic latitudes. We study the effect of upstream Alfvén Mach number $\left(M_{\mathrm{A}}\right)$ and magnetic local time (MLT) on the speed ratio $V / V_{\mathrm{SW}}$. The comparison with theory is good. Namely, (i) flow speed ratios above unity occur behind the dawn-dusk terminator, (ii) those below unity occur on the dayside magnetosheath, and (iii) there is a good general agreement in the dependence of the $V$ ratio on $M_{\mathrm{A}}$.
\end{abstract}

Keywords. Magnetospheric physics (Magnetosheath)

\section{Introduction}

Accelerated flows at the magnetopause or its boundary layers have traditionally received attention because they can provide a compelling observational confirmation of the occur- rence of magnetic reconnection between the magnetosphere and the magnetosheath magnetic fields, as first postulated by Dungey (1961) (see, e.g., Paschmann et al., 1979; Sonnerup et al., 1981; Gosling et al., 1982).

Plasma acceleration can also occur inside the magnetosheath close to, but outside, the magnetopause. These accelerated flows are not due to reconnection but are rather the result of the draping of the interplanetary magnetic field (IMF) around the magnetosphere (e.g., Chen et al., 1993; Lavraud et al., 2007, 2013; Rosenqvist et al., 2007; Petrinec at al., 1997; Lavraud and Borovsky, 2008; Harris, 2011). As explained below, a characteristic feature of these events, which is useful in their identification, is that the flow tends to be perpendicular to the local magnetic field. This is in sharp contrast to accelerations due to magnetic reconnection, where the flow has a large field-aligned component.

For such an important subject, the documented examples of draping-related accelerated flows are at present few in number. Here we try to bridge this gap somewhat by a systematic survey of data coverage lasting over several years. Our aim here is to establish a basis for observation of these events by formulating a methodology, and producing a data set that may be useful in future studies of this subject. We identify 34 examples of ion accelerations in the magnetosheath that are likely the result of IMF draping around the magnetosphere. We also carry out a statistical analysis to determine global features of these accelerations, with focus mainly on magnetosheath speeds that exceed the speed of the solar wind. A statistical survey of global magnetosheath speeds for speeds smaller than the solar wind is undertaken by Lavraud et al. (2013). 
In hydrodynamical models of the flow around an obstacle (e.g., Spreiter et al., 1966), the flow speed in the magnetosheath never exceeds the solar wind speed. This is because magnetic forces on the flow are neglected since the magnetic field is introduced via the frozen-in condition after the solution of the flow problem has been obtained. But in a magnetohydrodynamic (MHD) treatment, and when the IMF is northward-pointing, the magnetic field is typically enhanced near the magnetopause, and the density and plasma $\beta$ decrease in a layer of thickness proportional to $1 / M_{\mathrm{A}}^{2}$ (Lees, 1964; Zwan and Wolf, 1976; Erkaev, 1988; Phan el al., 1994; Farrugia et al., 1995). This is called the plasma depletion layer/magnetic barrier (PDL), observational evidence for which was first advanced by Crooker et al. (1979) and Paschmann et al. (1978). Sonnerup (1976) pointed out that the flow in this layer tends to align itself to become more perpendicular to the magnetic field, and he called the resulting pattern "stagnation line" flow, as opposed to stagnation point flow.

We shall thus compare the observations and statistical trends with a MHD theory proposed recently (Erkaev et al., 2011, 2012). These authors used a semi-analytical approach to examine the magnetic and hydrodynamic forces due to draping for IMF $B_{z}$ north. This theory predicts accelerations due to draping globally on the surface of the magnetopause for a given upstream $M_{\mathrm{A}}$ that reach up to values of $60 \%$ greater than the speed of the solar wind. The highest speeds are reached on the nightside. This result was also independently obtained by global MHD modeling (Lavraud et al., 2007), and through observation (Lavraud et al., 2007; Rosenqvist et al., 2007).

\section{Methodology}

As a primary data set for the interplanetary observations, we use the Richardson and Cane (2010) interplanetary coronal mass ejection (ICME) list covering the period 19972010, examining magnetopause crossings made by the Geotail, Cluster, and THEMIS spacecraft during the northwardpointing phase of the ICMEs listed there. ICMEs and MCs were chosen because of their strong magnetic field and characteristically low Alfvén Mach number in comparison to the average solar wind (Farrugia et al., 1995; Lavraud and Borovsky, 2008; Lavraud et al., 2013). Further, the $B_{z}$ north phase of ICME/MC passage at Earth tends to last for many hours, thus increasing the possibility of having spacecraft observations in the right locale during such conditions. We have also include other events from year 2010.

We present here a nonexclusive data set of IMF field line draping events during this time. Although many more accelerations were observed than are noted here during this period, these events have not been included because they did not satisfy the guidelines set forth by our identification methodology.
Table 1. Some important parameters that distinguish between draping- and reconnection-related ion accelerations in the GSM equatorial plane. $\theta_{\mathrm{bv}}$ is the angle between the local $\boldsymbol{B}$ and $\boldsymbol{V}$ vectors. $V_{\mathrm{sw}}$ is the speed of the solar wind.

\begin{tabular}{lllc}
\hline & $\theta_{\mathrm{bv}}$ & Layer & $\begin{array}{c}\text { Acceleration } \\
\max \end{array}$ \\
\hline Draping & $\approx 90^{\circ}$ & magnetosheath & $1.6 \times V_{\mathrm{sw}}$ \\
Reconnection & $\approx 180^{\circ}, 0^{\circ}$ & $\begin{array}{l}\text { LLBL /Alfvén } \\
\text { speed based on }\end{array}$ & \\
& & magnetosphere & \\
& & parameters & \\
\hline
\end{tabular}

Our approach for identifying accelerations due to draping is a combination of techniques used by Lavraud et al. (2007) and Rosenqvist et al. (2007). First, we choose events for which the possibility of reconnection on the dayside magnetopause was minimized, and thus focus mainly (with few exceptions) on an IMF clock angle $<60^{\circ}$. Second, we try to exclude accelerations observed high off the GSM equatorial plane where it is possible to observe a flow burst due to reconnection poleward of the cusp, particularly when the spacecraft is located tailward of the terminator. Third, we calculate the flows parallel and perpendicular to the local magnetic field. Flows parallel to the magnetic field lines are often characteristic of reconnection-induced flow bursts that occur in the low-latitude boundary layer (LLBL) earthward of the magnetopause (e.g., Rosenqvist et al., 2007). By contrast, as explained in the Introduction, flows perpendicular to the magnetic field lines tend to be associated with IMF line draping. Fourth, we use the magnetic field parameters in conjunction with the plasma temperature and density as well as the ion and electron energy flux distributions to identify the magnetopause and surrounding regions.

To understand the difference between reconnection and IMF draping accelerations, a few parameters of interest when studying these flows are shown in Table 1.

\section{Observations}

Here we present three case studies illustrating a few important features observed from the events included in our statistics. First, we show a straightforward example of a draping event that has a well-defined magnetopause, and clear draping signatures in the nearby magnetosheath. Second, we show a double entry into the magnetosheath, each associated with a draping-related acceleration. Finally, we show a unique example of a skimming orbit where the spacecraft observes an acceleration on the boundary of the magnetopause for a very long time compared to the length intervals where this behavior is generally observed. 


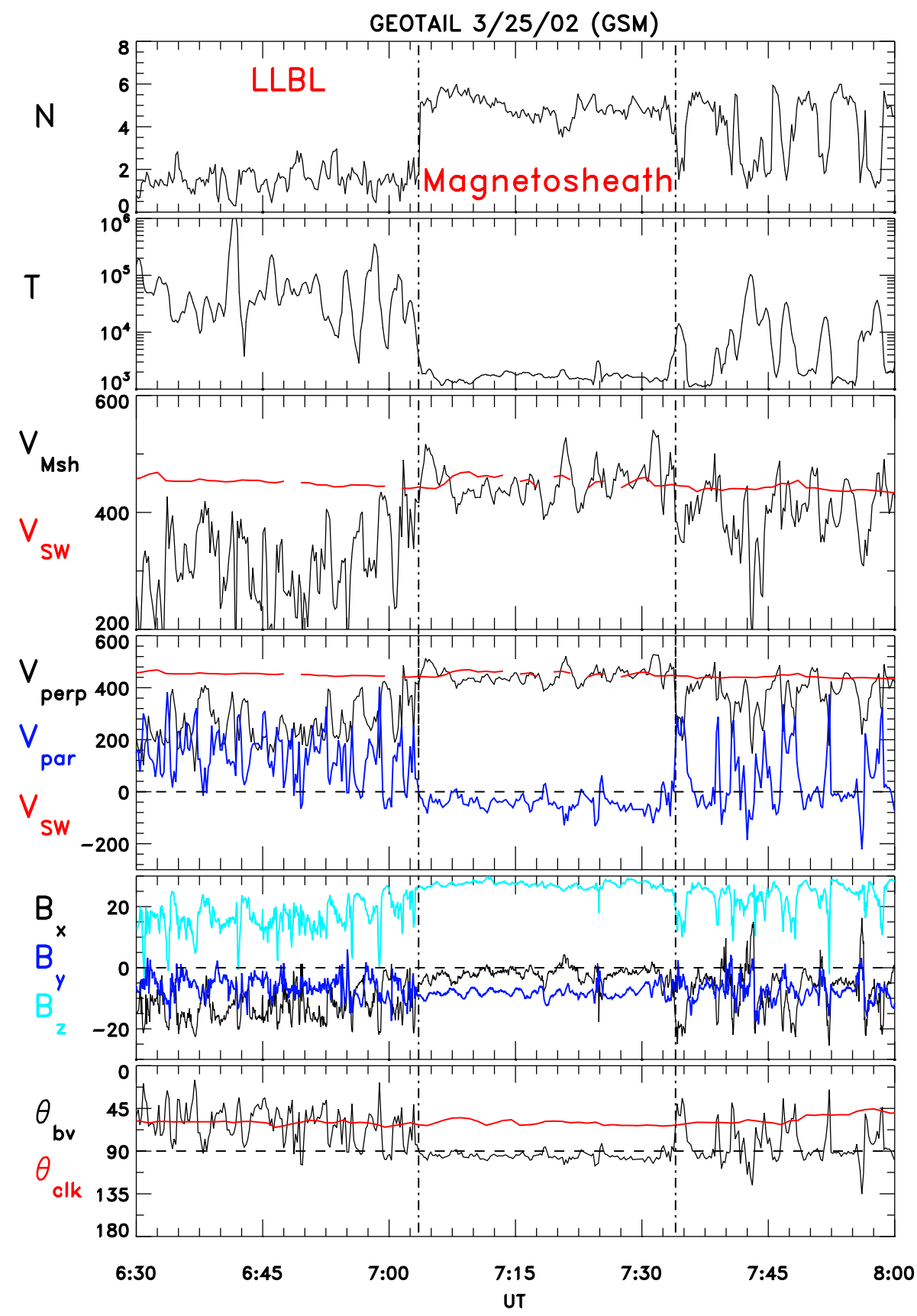

Fig. 1. Event on 25 March 2002. From top to bottom: the proton density in $\mathrm{cm}^{-3}$, proton temperature in $\mathrm{K}$. $V_{\mathrm{Msh}}$ and $V_{\mathrm{sw}}$, in $\mathrm{km} \mathrm{s}{ }^{-1}$, are the magnetosheath and solar wind speeds, respectively. $V_{\text {par }}$ and $V_{\text {perp }}$ are, respectively, the flow velocities parallel and perpendicular to the local magnetic field. The Geocentric Solar Magnetospheric (GSM) components of the magnetic field $B x, y, z$ are measured in nT. $\theta_{\mathrm{bv}}$ is the angle between the local magnetic field and the ion velocity. $\theta_{\text {clk }}$ is the IMF clock angle, i.e., the polar angle in the GSM $Y Z$ plane.

\subsection{Event 1 - Geotail 25 March 2002}

To ensure that our methodology is effective, we will now apply it to a more straightforward instance of IMF field line draping where there is little ambiguity about the location of the magnetopause. In Fig. 1 we show data from an outbound passage of Geotail through the LLBL into the magnetosheath on 25 March 2002. From top to bottom, the panels show the proton density, temperature, and bulk speed (in red, the solar wind speed); the ion flows perpendicular (black) and parallel (blue) to the magnetic field; the solar wind speed (red); the color-coded magnetic field components; and, in the bottom panel, the angle between the flow and field vectors (black) and the IMF clock angle (i.e., the polar angle in the 
GSM YZ plane: in red). The interplanetary data are from the ACE spacecraft in halo orbit around the L1 Lagrangian point. These have been shifted by the convection delay, $\Delta$, defined by the spacecraft separation in the $X$ direction divided by the $X$ component of the solar wind velocity.

At 07:12 UTC (first vertical guideline), Geotail is located at $(-11.69,-16.64,-1.52) R_{\mathrm{E}}$ (GSM coordinates), i.e., at low GSM latitudes tailward of the dawn terminator. During the period between the vertical guidelines, Geotail is in the magnetosheath, as inferred from the high densities and low temperatures. The clock angle of the IMF throughout much of the time Geotail is in the magnetosheath is $55^{\circ}$ (bottom panel).

Geotail crossed into the magnetosheath at 07:03 UTC. In contrast to the preceding LLBL traversal, the density here is high and the ion temperature low. Note the strongly northward field orientation (panel 5). The steady situation in the magnetosheath lasts up to 07:35 UTC, so the event is $\sim 32$ min long. From panels 3 and 4 it is seen that the flow of high-density plasma just tailward of the dawn terminator at low latitudes in the magnetosheath reaches and overtakes the solar wind speed for almost $1 / 2 \mathrm{~h}$. It is directed (panels 4 and 6) very close to perpendicular to the magnetic field.

This event is an easily identified example of IMF draping because of three major factors mentioned previously. Namely, (i) it is in agreement with all our initial requirements (low off the GSM YZ plane, and for low IMF clock angle), (ii) it occurs outside of the magnetopause, and (iii) the bulk ion flow during the acceleration occurs perpendicular to the local magnetic field. We know that the magnetopause is crossed at 07:03 UTC at the first vertical guideline, where a sharp increase in density occurs at the same time as a steep decrease in temperature and a change in magnetic field parameters.

The ion plasma flow from the Low Energy Particle (LEP) instrument on Geotail (Mukai et al., 1995) reached a maximum speed $20 \%$ greater than the speed of the solar wind, as is evident from panel 3. We are sure this was not the result of a short passage into the solar wind because of the proximity of this flow to the magnetopause and the steady values of the solar wind dynamic pressure at ACE (not shown).

The flow is perpendicular to the magnetic field $\left(\theta_{\mathrm{bv}} \approx 90^{\circ}\right)$ and extends throughout the entirety of Geotail's time in the magnetosheath. Geotail's orbit practically skims the magnetopause: the spacecraft is at $(-12.82,-16.32,3.46) R_{\mathrm{E}}$ and $(-11.31,-16.39,3.23) R_{\mathrm{E}}$ (GSE coordinates) at $06: 24$ and 08:00 UTC, respectively. That is, while tailward of the terminator it moves predominantly in the $+X$ direction. So this flow pattern is indicative of an extended stagnation line flow downtail of the terminators $\left(X_{\mathrm{gsm}}<0\right)$.

\subsection{Event 2 - Geotail: 13 April 1998}

While on an inbound pass on 13 April 1998, Geotail observed a double acceleration event during the time inter- val 05:15-06:00 UTC when the spacecraft was located at $(-18.5,19.9,6.0) R_{\mathrm{E}}$, which is plotted in Fig. 2. Thus the event occurs tailward of the dusk terminator but a few $R_{\mathrm{E}}$ deeper down the nightside than the previous example. Although the plasma parameters appear to fluctuate, there are two distinct crossings from the LLBL into the magnetosheath between the vertical guidelines shown, where the magnetic field changes, the density increase, and temperature decrease occur in step with each another. Reconnection is very unlikely to occur given the latitude and the solar wind conditions prevailing during the time of these crossings, as the two accelerations seen here occur at IMF clock angles between 1 and $48^{\circ}$. The maximum acceleration observed at each of the two magnetosheath crossings reaches a value of $28 \%$ higher than the speed of the solar wind.

The interplanetary cause of the double entry of Geotail into the magnetosheath is most likely a two-humped density (and hence dynamic pressure) profile seen at Wind and starting at 05:15 and 05:42 UTC, respectively. The Wind data are shown in Fig. 3. During this time Wind was located at (223.0, $-15.8,10.7) R_{\mathrm{E}}$, i.e., close enough to the Sun-Earth line for the measurements to represent those affecting the magnetopause after an appropriate delay time. Using the Geotail location and a $V_{x} \approx 406 \mathrm{~km} \mathrm{~s}^{-1}$, the delay from Wind to Geotail is $\sim 63 \mathrm{~min}$. In the figure the UTC at Wind has been shifted forward by this delay time. The times of the two dynamic pressure humps at Wind (panel 4) agree well with the times of the two excursions by Geotail into the magnetosheath. The temporal separation of the encounters at Wind and at Geotail supports this interpretation.

\subsection{Event 3 - Geotail: 20 August 2006}

This particular event is of great interest given the length of time $(\sim 1.75 \mathrm{~h})$ that Geotail observes accelerated flows, the peak of which reaches values of $27 \%$ above the speed of the solar wind. Geotail is following an inbound trajectory, crossing the magnetopause tailward of the dusk terminator. Its position at 10:00 UTC is $(-14.5,22.3,-6.3) R_{\mathrm{E}}$. Observations in the time interval 10:00-13:00 UTC are plotted in Fig. 4. Flow speeds exceeding the solar wind speed are seen from 10:00 to 11:45 UTC, before the first crossing into the LLBL, and in the second magnetosheath stay from 11:52 to 12:12 UTC, under a strongly northward IMF. The flows are directed mainly perpendicular to the local magnetic field (fourth panel). The increase in proton density centered around 11:20 UTC is not due to any transition region between the magnetosheath and the magnetosphere. Rather, it is likely the result of a density peak observed by ACE at 10:16 UTC (not shown). 


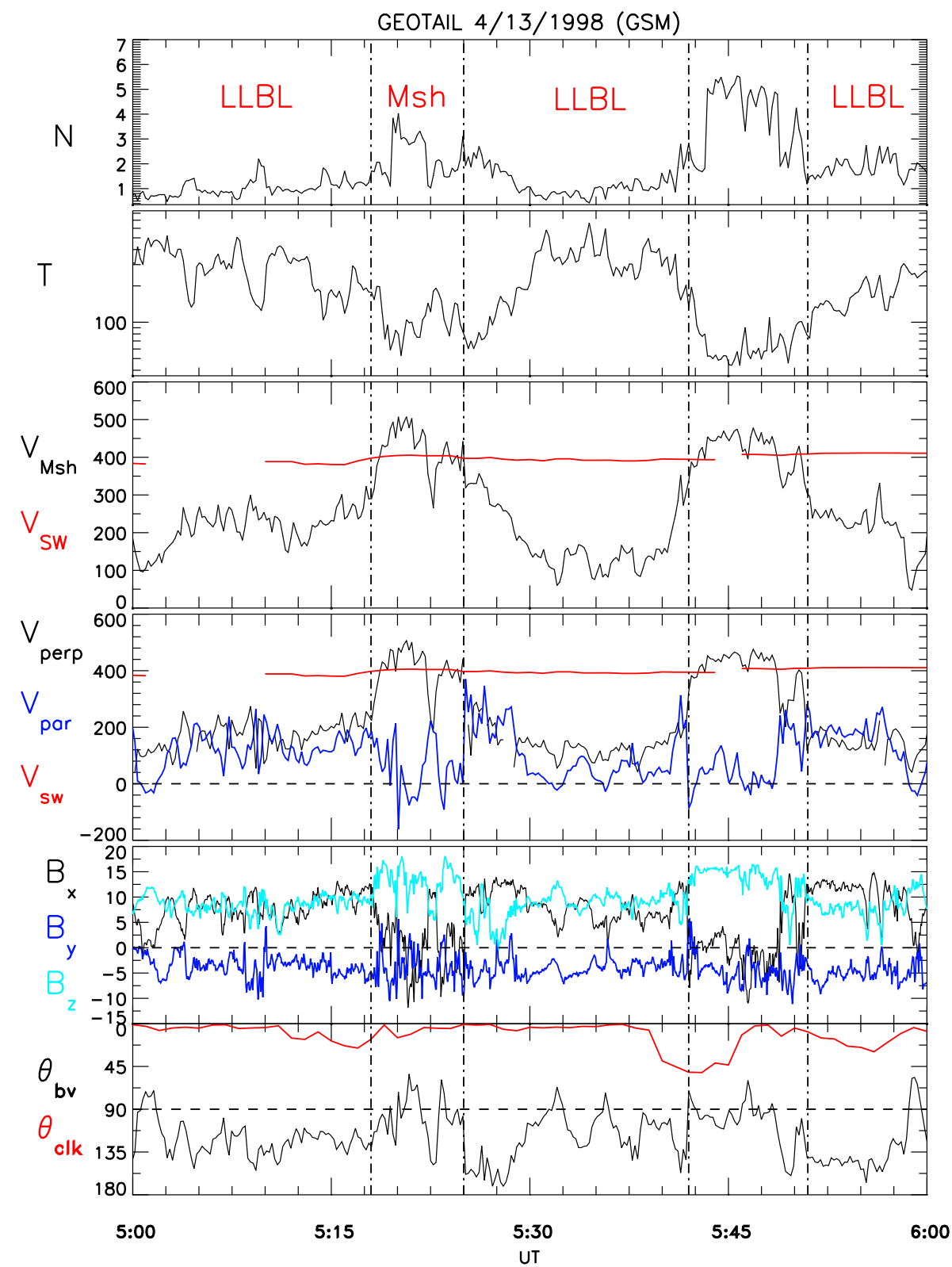

Fig. 2. Event on 13 April 1998. Two crossings into the magnetosheath are shown between pairs of vertical guidelines where the maximum sheath speed exceeds that of the solar wind.

A long-lasting event is presented by Rosenqvist et al. (2007) where the duration of the accelerated flow is on the order of $15 \mathrm{~min}$. These are exceptional examples, considering that most accelerations we have studied are on the order of 2-5 min. These accelerations are often short-lived because they are identified next to the magnetopause where the draping effect is strongest, and the probability of a spacecraft skimming the magnetopause during a low- $M_{\mathrm{A}}$ period (where the plasma depletion layer increases in size) is decidedly low. Geotail crosses the magnetopause at $(-15.1,21.3$, -6.3 ) (GSE).

\section{Statistical results: comparison with theory}

As magnetic field lines drape over the magnetosphere, they are stretched and distorted. This process is commonly referred to as field line draping. This draping effect creates a tension force on the magnetic field lines that, together with the total pressure gradient force, causes the local plasma that carries the field line around the magnetosphere to be accelerated. The steady state MHD momentum equation is given by

$$
\rho \boldsymbol{v} \cdot \nabla \boldsymbol{v}=\boldsymbol{J} \times \boldsymbol{B}-\nabla \boldsymbol{P}=\boldsymbol{F}_{\mathrm{B}}-\nabla \Pi \text {. }
$$




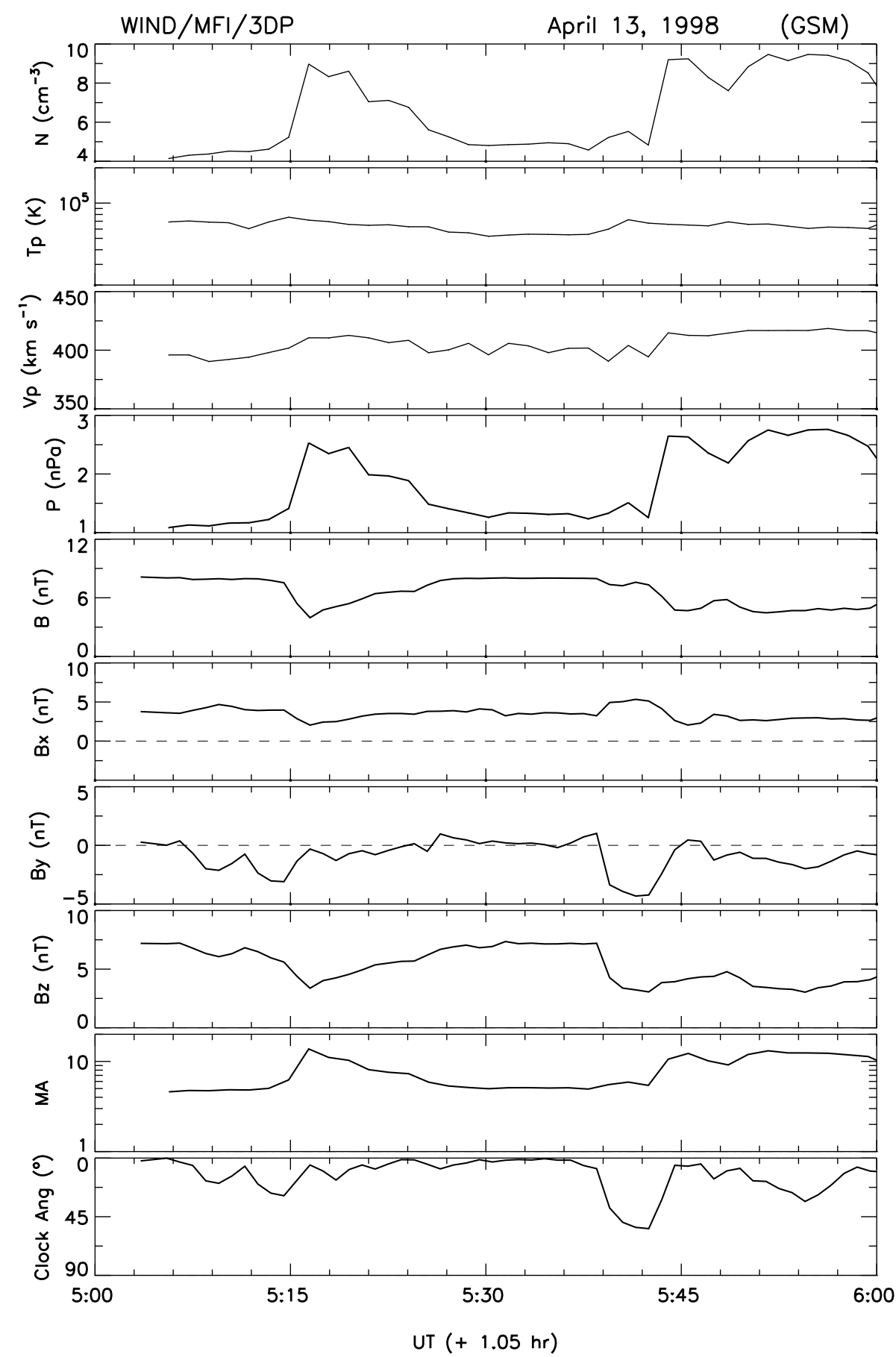

Fig. 3. Interplanetary data from Wind for the event on 13 April 1998: proton density, temperature, bulk speed, dynamic pressure, GSM components of the magnetic field, total field strength, Alfvén Mach number, and IMF clock angle.

The Lorentz force and the gradient of the thermal plasma pressure have been expressed as the sum of the magnetic curvature force $\left(\boldsymbol{F}_{\mathrm{B}}\right)$ and the gradient of the total pressure. The physical mechanism in which these two forces contribute to the acceleration of magnetosheath plasma has been explained by Farrugia et al. (1995), Lavraud et al. (2007, 2013), and
Erkaev et al. (2011, 2012), to which we refer the reader for further details. This is in contrast with reconnectionrelated accelerations that occur on the earthward side of the magnetopause.

In brief, we expect the total pressure gradient force on the dayside of the magnetosphere to reinforce the magnetic 


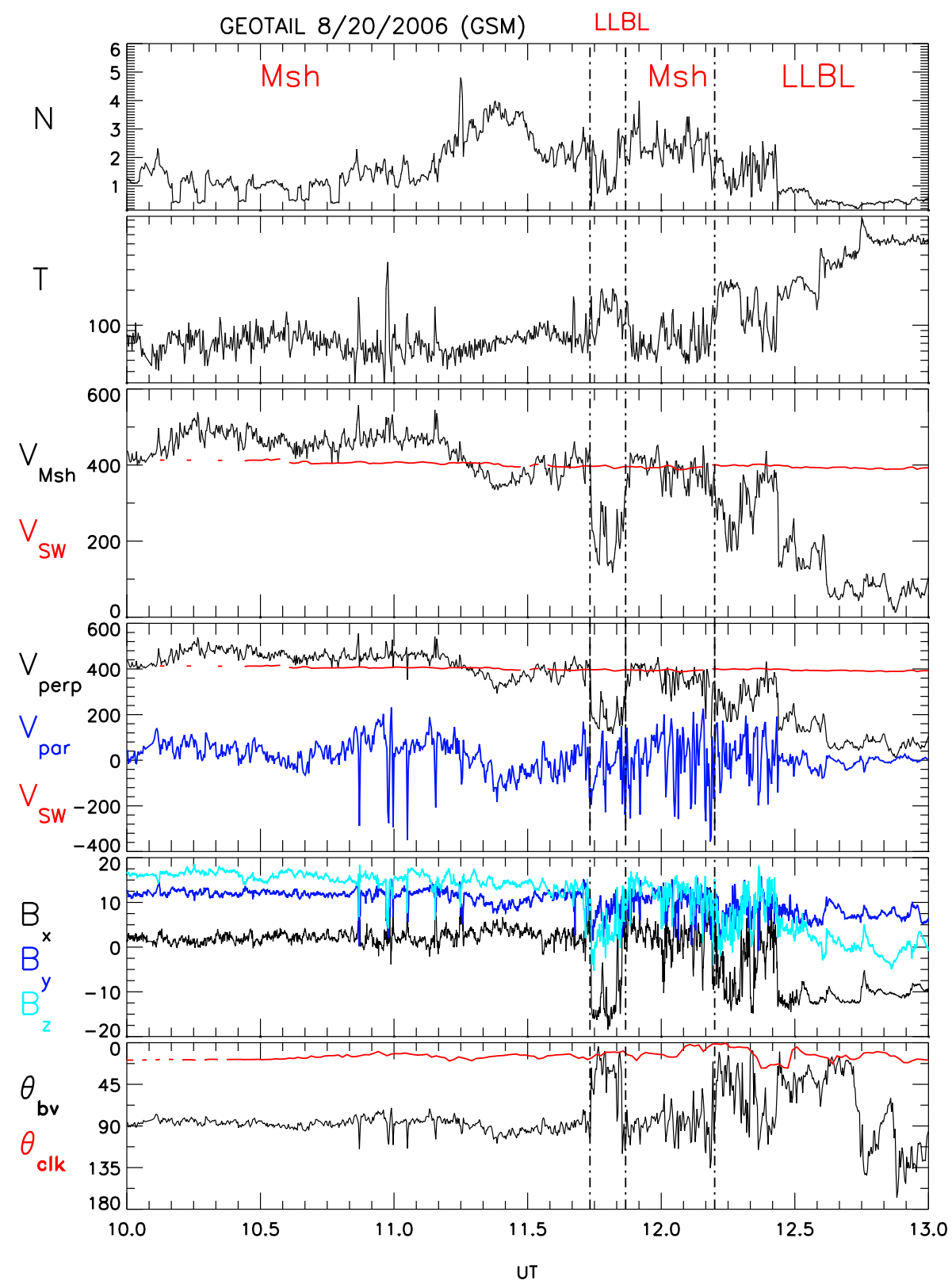

Fig. 4. Event on 20 August 2006. Same format as in Fig. 3. Notice the parameter $\theta_{\mathrm{bv}}$, which is plotted at the bottom with the clock angle of the IMF $\theta_{\mathrm{clk}} . \theta_{\mathrm{bv}}$ is practically constant at $90^{\circ}$ for $1.75 \mathrm{~h}$ in the magnetosheath leading up to the first magnetopause crossing at $11: 45 \mathrm{UTC}$.

curvature force because they point in the same direction (tailward), causing the magnetosheath particles to accelerate tailward (Chen et al., 1993; Lavraud et al., 2007). Near the terminators, this magnetic curvature force can reverse direction and oppose the total pressure gradient force depending on the shape of the magnetosphere assumed. (We use the Shue et al., 1997, magnetopause shape.) Far downstream of the terminators on the magnetopause, this change in direction of the curvature force will reduce the acceleration until the net force is zero. The maximum plasma speed due to draping is reached when the net force vanishes, which Erkaev et al. (2011, 2012) predicted should occur a few $R_{\mathrm{E}}$ tailward of the terminators.

Table 2 is a list of acceleration events, which represents a nonexclusive survey of the Richardson and Cane ICME list from 1997 to 2009 for Geotail, Cluster, and Themis, and events in 2010. Previously, there were only a handful of events ever suggested to be related to draping, so this set of 34 events represents many useful examples of IMF draping accelerations. 
Table 2. Event list.

\begin{tabular}{|c|c|c|c|c|c|c|c|c|c|c|c|}
\hline Day & Mo. ${ }^{1}$ & Year & Time & $V_{\mathrm{msh}} / V_{\mathrm{sw}}^{2}$ & $\mathrm{MA}_{\mathrm{sW}}^{3}$ & $\operatorname{Pdyn}_{\mathrm{sw}}^{4}$ & $\mathrm{MLT}^{5}$ & $\mathrm{XGSM}_{\mathrm{msh}}^{6}$ & $\mathrm{ZGSM}_{\mathrm{msh}}$ & $\theta_{\mathrm{clk}(\mathrm{sw})}^{7}$ & $\mathrm{SC}^{8}$ \\
\hline 11 & 10 & 1997 & 14.47 & 1.31 & 3.75 & 1.42 & $03: 54$ & -11.02 & 6.86 & 57.2 & GE \\
\hline 22 & 11 & 1997 & 19.0 & 1.67 & 1.45 & 1.56 & 03:06 & -19.70 & -1.13 & 29.2 & GE \\
\hline 13 & 4 & 1998 & 5.33 & 1.34 & 4.63 & 1.08 & $20: 49$ & -18.37 & 6.20 & 27.4 & GE \\
\hline 13 & 4 & 1998 & 5.75 & 1.22 & 3.90 & 1.02 & $20: 51$ & -18.49 & 5.78 & 0.20 & GE \\
\hline 23 & 1 & 1999 & 12.82 & 1.14 & 5.93 & 4.98 & $02: 51$ & -20.43 & -0.92 & 57.0 & GE \\
\hline 26 & 5 & 2000 & 18.8 & 1.30 & 22.0 & 0.00 & $20: 38$ & -14.28 & 9.17 & 29.8 & GE \\
\hline 11 & 6 & 2000 & 18.04 & 1.13 & 8.75 & 3.08 & $20: 21$ & -12.11 & 6.02 & 39.1 & GE \\
\hline 12 & 4 & 2001 & 15.20 & 1.39 & 2.10 & 0.56 & $20: 21$ & -13.76 & 7.00 & 50.8 & GE \\
\hline 12 & 4 & 2001 & 15.54 & 1.16 & 2.20 & 0.58 & $20: 24$ & -14.03 & 7.44 & 52.4 & GE \\
\hline 12 & 4 & 2001 & 16.10 & 1.30 & 3.71 & 1.54 & $20: 28$ & -14.29 & 7.89 & 52.2 & GE \\
\hline 31 & 5 & 2001 & 7.33 & 1.40 & 3.94 & 0.43 & 20:08 & -14.86 & 4.96 & 55.0 & GE \\
\hline 25 & 11 & 2001 & 7.35 & 1.44 & 3.42 & 6.45 & 19:00 & -3.70 & -3.36 & 27.6 & CL \\
\hline 25 & 11 & 2001 & 8.43 & 1.29 & 2.41 & 8.51 & $19: 04$ & -3.46 & -4.78 & 29.4 & $\mathrm{CL}$ \\
\hline 25 & 11 & 2001 & 9.23 & 1.61 & 2.77 & 8.80 & 19:05 & -3.32 & 1.68 & 6.9 & CL \\
\hline 28 & 12 & 2001 & 22.1 & 1.06 & 9.68 & 3.92 & 02:59 & -16.98 & -3.24 & 32.9 & GE \\
\hline 19 & 1 & 2002 & 11.78 & 1.44 & 3.75 & 4.20 & 03:17 & -20.02 & 2.66 & 40.8 & GE \\
\hline 25 & 3 & 2002 & 7.52 & 1.21 & 2.85 & 2.04 & 03:39 & -11.69 & -1.52 & 62.8 & GE \\
\hline 20 & 5 & 2002 & 5.87 & 1.13 & 5.50 & 3.83 & $21: 13$ & -21.58 & 8.58 & 59.4 & GE \\
\hline 17 & 11 & 2002 & 5.19 & 1.31 & 5.63 & 2.87 & $19: 36$ & -5.66 & -5.45 & 55.0 & CL \\
\hline 17 & 11 & 2002 & 4.89 & 1.26 & 5.50 & 3.00 & $19: 33$ & -5.76 & -4.76 & 45.0 & CL \\
\hline 21 & 11 & 2003 & 2.73 & 1.28 & 4.29 & 4.07 & $19: 15$ & -3.81 & -5.72 & 31.7 & $\mathrm{CL}$ \\
\hline 20 & 8 & 2006 & 10.86 & 1.36 & 3.21 & 1.25 & $20: 20$ & -14.85 & -16.47 & 12.1 & GE \\
\hline 19 & 11 & 2006 & 18.54 & 1.26 & 4.24 & 0.91 & $19: 17$ & -3.47 & -11.33 & 62.1 & CL \\
\hline 19 & 11 & 2006 & 19.84 & 1.20 & 4.45 & 0.96 & $19: 14$ & -3.03 & -11.01 & 59.6 & CL \\
\hline 3 & 12 & 2006 & 13.5 & 1.03 & 16.4 & 1.40 & $19: 03$ & -2.60 & -8.39 & 79.6 & CL \\
\hline 14 & 2 & 2007 & 19.9 & 0.88 & 10.0 & 2.08 & $13: 43$ & 8.95 & 5.19 & 67.5 & CL \\
\hline 21 & 6 & 2009 & 11.9 & 1.28 & 5.05 & 0.63 & $05: 27$ & -6.54 & -7.61 & 79.0 & CL \\
\hline 27 & 6 & 2009 & 12.19 & 1.14 & 12.1 & 1.07 & 06:04 & 0.28 & 0.05 & 29.8 & CL \\
\hline 12 & 12 & 2009 & 17.21 & 1.20 & 8.07 & 1.80 & $19: 11$ & -3.46 & -6.99 & 44.2 & $\mathrm{CL}$ \\
\hline 12 & 12 & 2009 & 17.70 & 1.15 & 7.80 & 1.80 & 19:08 & -3.32 & -6.85 & 75.0 & $\mathrm{CL}$ \\
\hline 4 & 1 & 2010 & 4.95 & 1.15 & 12.5 & 2.80 & $06: 31$ & 2.08 & -0.11 & 80.8 & $\mathrm{CL}$ \\
\hline 3 & 3 & 2010 & 6.15 & 0.91 & 14.2 & 2.01 & $11: 10$ & 9.99 & -8.70 & 85.0 & CL \\
\hline 1 & 4 & 2010 & 16.9 & 0.96 & 12.6 & 1.50 & 09:53 & 5.70 & -9.76 & 69.8 & CL \\
\hline 3 & 4 & 2010 & 23.0 & 0.93 & 9.37 & 2.28 & $10: 10$ & 5.56 & -10.72 & 8.5 & $\mathrm{CL}$ \\
\hline
\end{tabular}

${ }^{1}$ Mo. $=$ month, ${ }^{2} V_{\mathrm{msh}} / V_{\mathrm{Sw}}=$ magnitude of velocity measured in the magnetosheath divided by the velocity magnitude in the solar wind, ${ }^{3}$ MA $\mathrm{sw}_{\mathrm{SW}}=$ the Alfvén Mach number of the solar wind, ${ }^{4} \mathrm{Pdyn}_{\mathrm{sw}}=$ dynamic pressure of the solar wind, ${ }^{5} \mathrm{MLT}=$ magnetic local time of the spacecraft, ${ }^{6} \mathrm{XGSM}_{\mathrm{msh}}=X$ position of the spacecraft in the magnetosheath, ${ }^{7} \theta_{\mathrm{clk}(\mathrm{sw})}=$ clock angle of the IMF measured by the spacecraft upstream of the Earth's bow shock, ${ }^{8} \mathrm{SC}=$ spacecraft.

We now present our statistical analysis and compare the results with theory. Figure 5 shows a scatter plot of the maximum observed speed ratio versus $X$ expressed as a fraction of the subsolar radius of curvature, $L_{0}$, calculated from the Shue et al. (1997) model, for two contrasting ranges of the Alfvén Mach number, namely $M_{\mathrm{A}}$ in the range $[4,6]$ (low; black) and $M_{\mathrm{A}}>12$ (high; red). The plot here is effectively columns 5 and 9 of Table 2, calculated in terms of the Shue et al. (1997) subsolar radius of curvature, $L_{0}$. The higher Mach number points occurred both during higher $M_{\mathrm{A}}$ periods of the ICMEs and MCs, as well as during a broader period that was browsed in the search for transients. Error bars are excluded from the plots for visual clarity, because the sources of error were small. The greatest contributor to error was in the so- lar wind delay, which during an ICME with largely constant velocity is small, and for our cases was $<5 \%$.

The following points may be made: (i) for both sets the highest speed ratios are reached about $15 R_{\mathrm{E}}$ tailward of dawn-dusk terminator. (ii) For the smaller $M_{\mathrm{A}}$ range the speed ratio is higher. These observations are in general agreement with the theory of Erkaev et al. (2011). We use a polynomial fit for this plot since the curves in Erkaev et al. (2011) are obtained through numerical integration.

In Fig. 6 we plot the ratio of the maximum observed ion velocity compared to that of the solar wind as a function of $M_{\mathrm{A}}$. Overlaid in blue in Fig. 6 is the theoretical maximum velocity ratio for a given $M_{\mathrm{A}}$ as well as the curve of best fit for the observational points (red). For the latter we used a geometric fit of the form $y=a_{0} \cdot x^{a_{1}}+a_{2}$. 


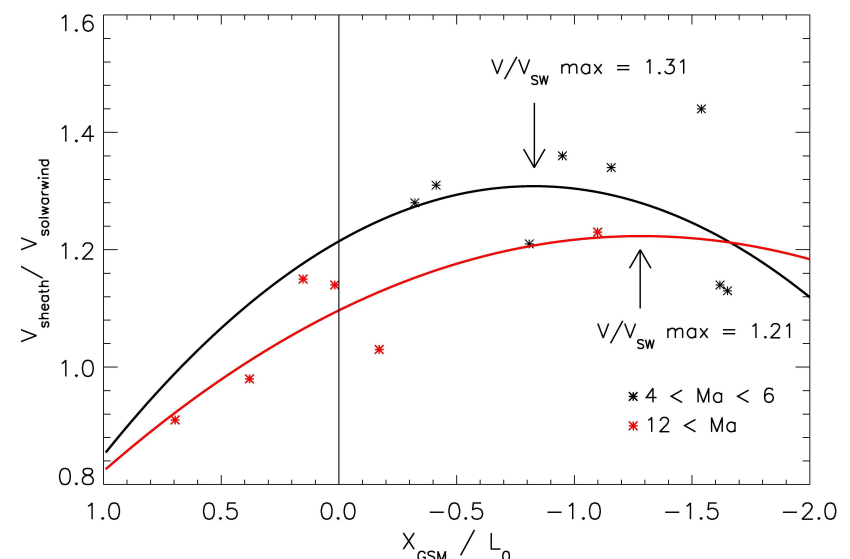

Fig. 5. A scatter plot of the speed ratio versus $X$ expressed as a fraction of the subsolar radius of curvature, $L_{0}$, for two ranges of $M_{\mathrm{A}}$. The black line is for $4<M_{\mathrm{A}}<6$, while the red line is for a much higher solar wind $M_{\mathrm{A}}$, namely $12<M_{\mathrm{A}}$, where the pressure gradient force is more dominant.

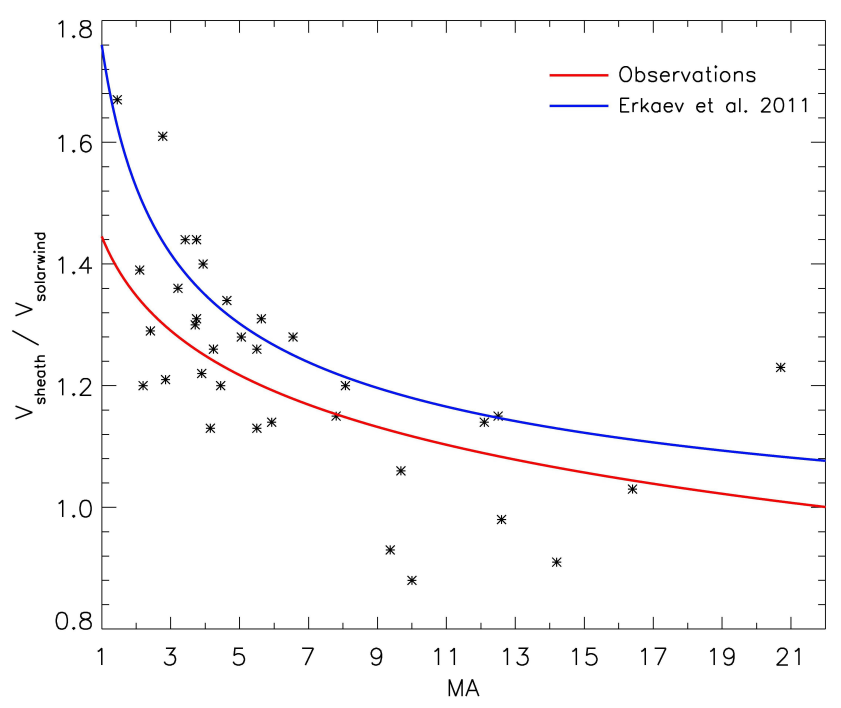

Fig. 6. The speed ratio predicted by Erkaev et al. (2011) (blue trace) against the fit to the observed data (red). A geometric fit of the form $y=a_{0} \cdot x^{a_{1}}+a_{2}$ is used for the latter. All MLTs are included, hence the scatter of points. The error bars are estimated to be small, so they are not plotted.

It is seen that the trends are very similar although there is a small vertical offset. The offset is expected because the theory predicts the maximum observable speed for a given position near the equatorial plane, which would be extremely difficult to reproduce observationally because of the different positions at which these accelerations are seen. Even so, the overall agreement is reassuring.

The speed ratios as a function of the magnetic local times (MLT) of the measurements are plotted in Fig. 7. This figure confirms the theory that these accelerated flows are below the solar wind speed on the dayside, and above the solar

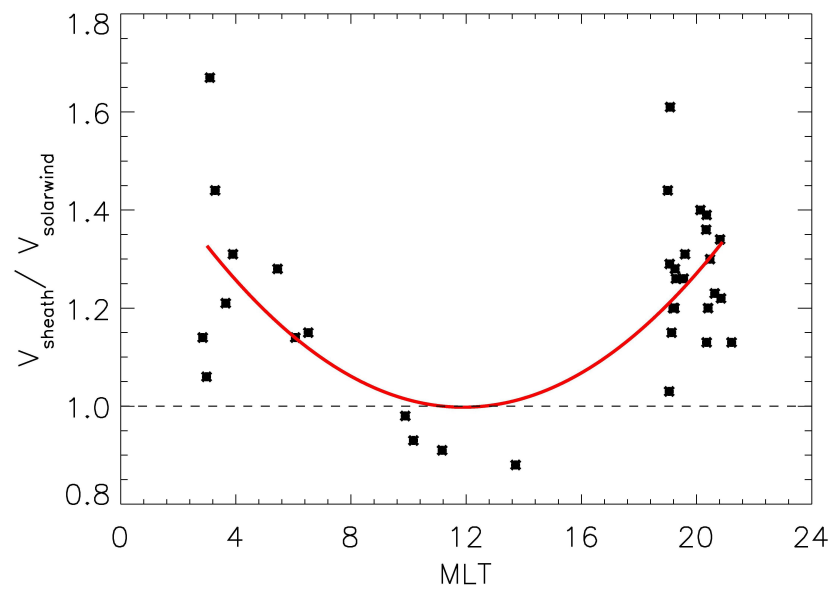

Fig. 7. This graph represents the ratio of the maximum magnetosheath speed compared to the solar wind speed for all $M_{\mathrm{A}}$ as a function of MLT. Note that the accelerations are smaller on the day side, and larger downtail of the terminators, as is suggested in the theory. Given the small number of points, this represents a semiqualitative representation of observations for all $M_{\mathrm{A}}$ and MLT.

wind speed on the nightside. A data gap exists in the MLT range 22:00-03:00 MLT, where none of the spacecraft sample the near-magnetopause magnetosheath. There is a second data gap in Fig. 7 from 15:00 to 18:00 MLT. The lack of data here is puzzling since we tended to find few events in this region despite looking for them explicitly. At present we are not certain of the origin of this gap.

\section{Discussion and conclusions}

In this paper, we have described three case studies of drapingrelated accelerated flows in the magnetosheath. We elaborated a data analysis approach to identify such events and distinguish them as far as possible from those due to reconnection, which are identified differently because of (i) their location inside the magnetopause and (ii) a significant flow component parallel to the magnetic field. We carried out statistical studies of average properties of these acceleration events based on a table of 34 events we identified. This compilation can help future work on an important subject where data examples have so far been few. Comparison with theory for IMF $B_{z}$ north was good: (i) in predicting higher flow ratios behind the dawn-dusk terminator, (ii) strongest accelerations at low latitudes, and (iii) general agreement of trends in the velocity ratio $V_{\mathrm{SW}} / V$ as a function of $M_{\mathrm{A}}$.

We have shown that magnetic field line draping is sufficient to explain the observed accelerations in the magnetosheath that are not related to reconnection. In this regard, our methodology of (i) identifying the magnetopause and associated boundary layer regions, (ii) ensuring that $\theta_{\mathrm{bv}} \approx 90$, and (iii) that the accelerated plasma was outside of the magnetopause and not connected to the magnetosphere has 
proved effective in making a distinction between bulk ion accelerations due to magnetic reconnection and those due to IMF draping.

After surveying the Richardson and Cane ICME list from 2007 to 2009 and looking for other events in 2010 for Geotail, Cluster, and Themis, we have determined that bulk ion accelerations in the magnetosheath that lead to speeds above that of the solar wind for $B_{z}$ north are relatively rare for flows that are unambiguously not related to reconnection. During most passages of spacecraft through the magnetosheath, however, it is not uncommon for an acceleration due to field line draping to be observed in the magnetosheath close to the magnetopause boundary. The likelihood that the acceleration will exceed the speed of the solar wind, however, is small. Here we focused, though not exclusively, on ICMEs or magnetic clouds (MCs) as interplanetary configurations because the associated long duration of northward $B_{z}$ in many of these transients is a favorable condition for identifying accelerated flows in the magnetosheath at low latitudes.

The three most important parameters in our study of IMF draping accelerations have been magnetic local time (MLT); the speed ratio, $V / V_{\mathrm{SW}}$; and $M_{\mathrm{A}}$. Using the Richardson and Cane ICME list and focusing on the northward phase of these magnetically dominated structures increases the probability of observing an increased size of the PDL because of the low $M_{\mathrm{A}}$ generally associated with ICMEs and because the width of the PDL increases with decreasing $M_{\mathrm{A}}$.

This process of field line draping is also valid for IMF south and/or pointing strongly in the $Y_{\mathrm{gsm}}$ direction. However, these examples have not been included in our data set (with the occasional clock angle $>50^{\circ}$ in Table 2) given the risk of inadvertently including a reconnection-related flow. The analysis presented here is sufficient to differentiate between reconnection and field line draping flows for IMF south as well, since the methodology to identify them is the same.

We showed cases where the accelerated flows lasted for a time on the order of $1 \mathrm{~h}$. Assuming steady conditions and an orbit across the magnetosheath, this implies a thickness of a few $R_{\mathrm{E}}$ for the regions where accelerations occur. This validates the conjecture of Lavraud et al. (2007) that the accelerated region can have a thickness of a few $R_{\mathrm{E}}$ normal to the magnetopause. These authors inferred this by triangulation during unsteady conditions. We did this by direct observations during steady interplanetary conditions. Further, we presented a case where Geotail was on an orbit that skimmed the magnetopause. This was an event of long duration $(1.75 \mathrm{~h})$. The accelerated flows were perpendicular $\pm 30^{\circ}$ to the field, as they should be.

We have emphasized accelerations leading to speeds faster than the solar wind. However, our events do include cases of enhanced flows that lie below the solar wind speed. These typically occur on the dayside, as also predicted by theory. Dayside accelerations in connection with PDLs forming at the dayside magnetopause for northward IMF have been re- ported in the survey of low shear magnetopauses by Phan et al. (1994) and from an AMPTE/UKS and IRM crossing of the magnetopause/magnetosheath also under northward IMF by Farrugia et al. (1998).

Acknowledgements. Work at UNH is supported by NASA Grants NNX10AQ29G and NNX13AP39G. N. V. Erkaev is supported by grant No. 12-05-00152-a from the Russian Foundation of Basic Research.

Topical Editor C. Owen thanks the two anonymous referees for their help in evaluating this paper.

\section{References}

Chen, S. H.,Kivelson, M. G., Gosling, J. T., Walker, R. J., and Lazarus, A. J.: Anomalous aspects of magnetosheath flow and of the shape and oscillations of the magnetopause during an interval of strongly northward interplanetary magnetic field, J. Geophys. Res., 98, 5727-5742, 1993.

Crooker, N. U., Eastman, T. E., and Stiles, G. S.: Observations of plasma depletion in the magnetosheath at the magnetopause, J. Geophys. Res., 84, 869-874, 1979.

Dungey, J.: Interplanetary magnetic field and the auroral zones, Phys. Rev. Lett., 6, 47-48, 1961.

Erkaev, N. V.: Results of the investigation of MHD flow around the magnetosphere, Geomagn. Aeron., 28, 455-464, 1988.

Erkaev, N. V., Farrugia, C. J., Harris, B., Biernat, H. K.: On accelerated magnetosheath flows under northward IMF, Geophys Res. Lett., 38, L01104, doi:10.1029/2010GL045998, 2011.

Erkaev, N. V., Farrugia, C. J., Mezentsev, A. V., Torbert, R. B., and Biernat H. K.: Accelerated magnetosheath flows caused by IMF draping: Dependence on latitude, Geophys. Res. Lett., 39, L01103, doi:10.1029/2011GL050209, 2012.

Farrugia, C. J., Erkaev, N. V., Biernat, H. K., and Burlaga, L. F.: Anomalous magnetosheath properties during Earth passage of an interplanetary magnetic cloud, J. Geophys. Res., 100, 1924519257, 1995.

Farrugia, C. J., Biernat, H. K., Erkaev, N. V., Kistler, L. M., Le, G., and Russell, T.: MHD model of magnetosheath flow: comparison with AMPTE/IRM observations on 24 October, 1985, Ann. Geophys., 16, 518-527, doi:10.1007/s00585-998-0518-7, 1998.

Gosling, J. T., Asbridge, J. R., Bame, S. J., Feldman, W. C., Paschmann, G., Sckopke, N., and Russell, C. T.: Evidence for quasi-stationary reconnection at the dayside magnetopause, J. Geophys. Res., 87, 2147-2158, doi:10.1029/JA087iA04p02147, 1982.

Harris, B.: Observational aspects of IMF Draping around the magnetosphere, Masters Thesis, University of New Hampshire, Durham, NH, 2011.

Lavraud, B. and Borovsky, J. E.: Altered solar wind-magnetosphere interaction at low Mach numbers: Coronal mass ejections, J. Geophys. Res., 113, A00B08, doi:10.1029/2008JA013192, 2008.

Lavraud, B., Borovsky, J. E., Ridley, A. J., Pogue, E. W., Thomsen, M. F., Réme, H., Fazakerley, A. N., and Lucek, E. A.: Strong bulk acceleration in the Earth's magnetosheath: A magnetic slingshot effect?, Geophys. Res. Lett., 34, L14102, doi:10.1029/2007GL030024, 2007. 
Lavraud, B., Larroque, E., Budnik, E., Génot, V., Borovsky, J. E., Dunlop, M. W., Foullon, C., Hasegawa, H., Jacquey, C., Nykyri, K., Ruffenach, A., Taylor, M. G., Dandouras, I., and Rème, H.: Asymmetry of magnetosheath flows and magnetopause shape during low Alfvń Mach number solar wind, J. Geophys. Res.Space, 118, 1089-1100, doi:10.1002/jgra.50145, 2013.

Lees, L. : Interaction between the solar wind plasma and the geomagnetic cavity, AAIA J., 2, 1576-1582, 1964.

Mukai, T., Machida, S., Saito, Y., Hirahara, M., Terasawa, T., Kaya, N., Obara, T., Ejiri, M., and Nishida, A.: The low energy particle (LEP) experiment onboard the Geotail satellite, J. Geomag. Geoelectr., 46, 669-692, 1994.

Paschmann, G., Sckopke, N., Haerendel, G., Papmastorakis, I., Bame, S. J., Asbridge, J. R., Gosling, J. T., Hones Jr., E. W., and Tech, E. T.: The ISEE plasma observations near the subsolar magnetopause, Space Sci. Rev., 22, 717-737, 1978.

Paschmann, G., Sonnerup, B. U. Ö., Papamastorakis, I., Sckopke, N., Haerendel, G., Bame, S. J., Asbridge, J. R., Gosling, J. T., Russell, C. T., and Elphic, R. C.: Plasma acceleration at the earth's magnetopause: Evidence for reconnection, Nature, 282, 243-246, 1979.

Petrinec, S. M., Mukai, T., Nishida, A., Yamamoto, T., Nakamura, T. K., and Kokubun, S.: Geotail observations of Magnetosheath flow near the magnetopause, using Wind as a solar wind monitor, J. Geophys. Res., 102, 26943-26959, 1979.

Phan, T. D., Paschmann, G., Baumjohann, W., Sckopke, N., and Lühr, H.: The magnetosheath region adjacent to the dayside magnetopause: AMPTE/IRM observations, J. Geophys. Res., 99, 121-141, 1994.
Richardson, I. G. and Cane, H. V.: Near-Earth Interplanetary Coronal Mass Ejection During Solar cycle 23 (1996-2009): Catalog and Summary of Properties, Solar Phys., 264, 189-237, doi:10.1007/s11207-010-9568-6, 2010.

Rosenqvist, L., Kullen, A., and Buchert, S.: An unusual giant spiral arc in the polar cap region during the northward phase of a Coronal Mass Ejection, Ann. Geophys., 25, 507-517, doi:10.5194/angeo-25-507-2007, 2007.

Shue, J. H., Chao, J. K., Fu, H. C., Khurana, K. K., Russell, C. T., Singer, H. J., and Song, P.: A new functional form to study the solar wind control of the magnetopause size and shape, J. Geophys. Res., 102, 9497-9511, 1997.

Sonnerup, B. U. O.: The reconnecting Magnetopause, in: Magnetospheric Physics, edited by: McCormac, B. M., D. Reidel, Norwell, Mass, 1976.

Sonnerup, B. U. O., Paschmann, G., Papamastorakis, I., Sckopke, N., Haerendel, G., Bame, S., Asbridge, J., Gosling, J., and Russell, C. T.: Evidence for Magnetic field reconnection at the Earth's magnetopause, J. Geophys. Res., 86, 10049-10067, 1981.

Spreiter, J. R., Summers, A. L., and Alksne, A. Y.: Hydromagnetic flow around the magnetosphere, Planet. Space Sci., 14, 223-250, 1996.

Zwan, B. J. and Wolf, R. A.: Depletion of the solar wind plasma near a planetary boundary, J. Geophys. Res., 81, 1636-1648, 1976. 